\title{
Neurological Registry Feasibility and Sustainability
}

\author{
Tamara Pringsheim ${ }^{1}$, Ruth Ann Marrie ${ }^{2}$, Elizabeth Donner ${ }^{3}$, \\ Michael Shevell ${ }^{4}$, Darren Lam ${ }^{l}$, Lundy Day ${ }^{l}$, Megan Johnston ${ }^{1}$, \\ Nathalie Jette $^{1,5}$, Lawrence Korngut ${ }^{1}$
}

Can J Neurol Sci. 2013; 40: Suppl. 2 - S55-S59

The feasibility and sustainability of a registry depend on many factors, including researchers, clinicians, administrators and participants. The development and maintenance of a successful registry may be improved by considering the following elements in the design and implementation of registry procedures.

\section{RELEVANT Literature}

\section{Factors That Negatively Affect Feasibility}

Several factors can negatively influence registry feasibility. They include confidentiality and privacy issues, barriers to participation, issues related to multiple centres and locations, issues related to human and financial resources, poor data quality(non-uniform, missing, or incomplete data), and potential biases.

In the past, concerns over maintaining privacy have led to patients declining to give personal / sociodemographic information, concealing their diagnosis, or even submitting false information. ${ }^{254}$ The ethical concerns surrounding participant consent, ${ }^{41,255}$ and privacy legislation ${ }^{39}$ can also complicate registries, especially when disagreement exists regarding whether individual privacy can be overridden for the greater public good for quality assurance projects. Differences in legislation across jurisdictions can add further complexity to the design of registries whose source populations are widely dispersed. Issues of confidentiality are not unique to patients; physicians have also been concerned about privacy. In one study, surgeons expressed concerns about who has access to patient outcomes data. ${ }^{256}$ Finally, concerns about security when recording and transmitting participant data online have been raised. ${ }^{257}$

Registries often depend on the participation of multiple stakeholders, and low participation and response rates by any stakeholder, including health care providers, institutions, and participants are problematic. Many physicians may be reluctant to participate if the commitment involves a large amount of time, effort, or money. ${ }^{53,256}$ Ensuring low physician burden by providing adequate financial and human resources to enable data collection may increase the likelihood of retaining physicians.
Anonymity at the physician level may also be important in the design of some registries. In one study, some surgeons declined to participate if they were fearful of revealing their outcomes when outcomes may appear worse than those of other surgeons. ${ }^{256}$ Troubles securing cooperation from hospitals and staff have also been reported..$^{254,258}$ For example, in establishing a statewide mammography database in Arkansas, potential participating mammography centres were concerned about patient confidentiality, lack of space, computers and staff time to orient employees on data collection procedures. Some centres' regulations prevented non-employee access to centre data and equipment ${ }^{258}$.

Overly demanding data collection requirements and the frequency of data collection may affect patient participation and retention. Several studies have reported that tools and forms that are not user-friendly may prohibit participation and proper functioning of a registry. ${ }^{36,103}$ Multimodal data collection (online, paper based) and tailoring the mode of data collection (e.g. support mobile application) to the needs of the participants may increase enrollment and retention. Registries may be more successful with respect to recruitment and retention if the patients have a relationship with people collecting data, and regular clinical follow up may help to enhance relationships. Members of some ethnic groups may be less willing to participate in registries than others. ${ }^{18,41,61}$ For example, in analyzing a large comprehensive data set of participants recruited into clinical research programs on Alzheimer's disease in the United States, only $3.6 \%$ of the total population studied was non-white ${ }^{61}$.

While some registries are limited to a single centre, many involve multiple sites, and obtain data via multiple sources. Multi-centre registries may face challenges due to inconsistent data collection methodology, which can limit completeness and data comparability. Definitions for a condition may differ by site, ${ }^{40,106,257,259}$ and reporting of outcomes may also differ. ${ }^{259}$ Conflict on policy development ${ }^{106}$ what data to collect, ${ }^{39}$ and differences in the availability of experts and resources ${ }^{42}$ may also arise. Data quality may differ as well due to non-uniform, missing, or incomplete datasets. As mentioned earlier, the lack of uniform definitions and data heterogeneity can lead to

From the ${ }^{1}$ Hotchkiss Brain Institute, University of Calgary, Calgary, Alberta; ${ }^{2}$ University of Manitoba, Winnipeg, Manitoba; ${ }^{3}$ University of Toronto, Hospital for Sick Children,

Toronto, Ontario; ${ }^{4} \mathrm{McGill}$ University, Montreal, Quebec; ${ }^{5}$ Institute for Public Health, University of Calgary, Calgary, Alberta.

Final Revisions Submitted January 28, 2013.

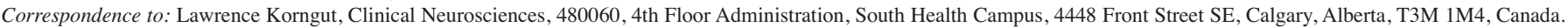

Email: Lawrence.korngut@albertahealthservices.ca. 
inconsistencies in data sets..$^{40,106,257,259}$ Fragmented data sets can also result from insufficient coverage of a population..$^{42,259}$

Other factors that can affect registry feasibility include financial and funding constraints, lack of time, effort, and resources, and potential biases. Insufficient financial support can critically limit the development and sustainability of registries. ${ }^{40,42,260}$ Studies have reported a need for trained, dedicated staff for the maintenance of a registry. ${ }^{39}$ Asking hospital staff to incorporate registry work into existing duties may not be sustainable and can lead to poor data quality. ${ }^{39}$ There may be a lack of interest and motivation with staff attending to the registry, especially when personnel turnover is high. ${ }^{258}$ This is also accompanied with the need to train new personnel that are unfamiliar with the registry. Others have documented a lack of resources such as staff (including experts in the field), space, computers, and time. ${ }^{42,258}$ Finally, selective reporting can result in selection biases that lead to biased results..$^{256,261}$

\section{Enabling Factors that Enhance Feasibility}

The literature outlines enabling factors that enhance the feasibility of registries including establishing a purpose that reflects the needs of registry users, adequate funding, consistent human resources, implementing a user friendly data entry process with a minimal data set and international collaboration when appropriate.

\section{Clear Purpose}

It is essential for a registry to have a predefined purpose that reflects the needs of its users. Having a predefined goal enables registry personnel to focus on a specific objective rather than simply collecting data. ${ }^{39}$ The purpose should be explicitly defined and agreed upon before the implementation of the registry, ${ }^{36}$ as along with explicit aims for data collection and usage. This includes planning and selection of data items to be included. ${ }^{39}$

\section{Data Collection}

Data collection should be focused based on the goals of the registry and limited to the data necessary to meet those goals. For registries that seek to gather data regarding many patients, limiting the data collection beyond that required to routine clinical care to five to ten minutes per patient encounter may increase the likelihood of ongoing participation. For registries that are gathering data involving small numbers of patients, it may be more feasible to gather larger amounts of data per encounter. A nurse or other allied health professional supported registry might require less physician resource time. Larger amounts of data collected increase the potential for inconsistent and missing data.

Several other factors must be considered during registry development including communication and organizational frameworks, infrastructure and $\operatorname{costs}^{41}$ and who should have access to participant information and for what purpose. ${ }^{256}$ It is also important to ensure the registry population will be large enough to support the conduct of valid scientific research and if using the registry design will best answer research questions. ${ }^{41}$

\section{Stakeholder Engagement}

For a registry to be sustainable it needs support from stakeholders at political, administrative and clinical levels. ${ }^{39}$ Active collaboration among researchers, policy makers, patient advocates and healthcare providers is important. ${ }^{40}$ Support for a registry can be influenced by establishing a steering committee, or expert panel. ${ }^{39}$ Steering committees help ensure timelines are met, objectives are clear, and that the interests of the general community are met. ${ }^{39}$ All stakeholders, including patient advocates, funding agencies, researchers and people involved in the operation of the registry should be considered for involvement in the steering committee. The level of enthusiasm and involvement of the steering committee, site champions, and principal investigators can determine the success or failure of the registry. Both ethical and scientific oversight committees can be established to address key issues related to registry design and implementation and make recommendations. ${ }^{41}$

\section{Communication}

Regular communication (e.g. teleconferences) from the data coordinating centre is vital to the success of the registry, by sustaining enthusiasm and a sense of purpose among participating centres. It is important to emphasize community building among the coordinating centre and participating sites and establish visibility at relevant national meetings. Registry sustainability can be enhanced by regular communication with the participating centres and site retention tools such as a website, newsletters, instruction manuals, training meetings, regular data reports, presentations at conferences, and the ability of participating providers to publish based on registry data. Centre specific data reports could be offered and this information may be attractive to centre representatives because it could enhance their institutional databases, or provide a means of quality assurance. Visible products should be clinically relevant to people contributing to data collection.

\section{Finances}

Financial feasibility must also be addressed when planning a registry. It is important to note that the scale of cost of a registry is not in a linear relationship with the scope of the registry. As the scope of a registry increases, the cost to implement further changes with the registry may rise at a greater rate than the change in scope. Ideally registry investment should be considered in the context of both benefit to society and minimized costs. The point at which the benefit to society equals the minimized cost is the ideal investment ${ }^{260}$ Adequate funding is needed to support ongoing data collection and quality assurance efforts. ${ }^{36,40,42}$ Requests for proposals for registry projects should have funding terms that are appropriate and meet the needs to fund a planning phase, execution phase, and analysis phase. It is important to be clear about what role sponsors have in registry planning and analysis, and who has access to data. Obtaining and sustaining funding requires a long-term commitment from an expert group, retention of experienced staff and attracting funding for additional research projects. ${ }^{39}$ Publication of registry results and other knowledge translation activities will improve the success of future applications for funding and may attract philanthropic funding sources. 


\section{Human Resources}

Registries need trained and skilled researchers and clinicians to coordinate, collect and analyze data. ${ }^{39,42} \mathrm{~A}$ full-time individual should be hired and trained to improve data quality. ${ }^{36}$ For example, to maintain long-term interest from collaborators, the Victorian State Trauma Registry aimed to train postdoctoral fellows and newly graduated specialists. ${ }^{39}$

\section{Change Management}

Proper change management is essential for registry success. ${ }^{5}$ It is recommended that registries have a manual which describes - in detail - policies, procedures, protocols, the governing body if the registries and the roles and responsibilities of its members, and processes and infrastructure for ongoing training of registry staff. $^{5}$ Furthermore, it is recommended to have in place a standard procedure for communicating about change in a timely manner. ${ }^{5}$

\section{Data Collection Practices that Promote Feasibility}

Depending on the goal of the registry, data collection should be population-based to provide unbiased data and enable monitoring and evaluation of the entire health care system. ${ }^{39}$ Mandatory participation by centre, where feasible, increases efficiency and accuracy of outcome results. ${ }^{256}$ Depending on the ethical and social landscape of the population, a combination of ascertainment methods may be required to include underrepresented groups. ${ }^{41}$

\section{Minimum core dataset}

There is a need for a minimum core data set that is complete enough to fulfill the purpose of registry, but limited enough to ensure feasibility and high quality of the data collected..$^{36,106}$ From a clinical perspective all data items should be included, but from an epidemiological perspective more data collected reduces the focus on data quality and completeness; these perspectives need to be balanced to meet the goals of the registry and ensure data quality. ${ }^{39}$ To limit heterogeneity of data, a consensus around core data elements should be developed. ${ }^{257}$ It is helpful to reevaluate the data items annually for completeness and relevance and to refine the data collection tool if needed. ${ }^{39}$ Data accuracy and completeness should be monitored regularly at all participating sites. ${ }^{36,40}$

\section{Data entry}

The data entry process should be user friendly, involving easy to use data entry forms with straightforward, universally accepted definitions and a focused data collection strategy. ${ }^{36,39,40,103}$ It is recommended that data collection be standardized, easy to access, reported regularly and entered without requiring interpretation. ${ }^{39,106}$ Online data submission via a secure web-based system can ease data reporting, ${ }^{103,259,262,263}$ but requires enhanced efforts to protect confidentiality of the data. ${ }^{103,259}$ Data quality can be improved by using clear coding guidelines, proper instructions for data collectors and using patient identifiable data for data linkage and validation. ${ }^{111}$ Pilot testing of registry elements prior to recruitment and the use of an advisory board to add transparency and credibility are good practices to ensure registry burden is minimized.

\section{Consent}

Linkage to other data sources in order to obtain key long term outcomes data on patients who are lost to follow-up may be helpful, such as vital statistics to determine whether the patient has died. Such linkages would need to be included in the consent form. It is beneficial to seek permission for future direct contact (without the consent/intervention of any associated health care providers) at the time of enrollment. Consent forms can be developed providing the option of participating, including data linkage and permission for future contact. Providing yes and no options for each choice allows participation to be tailored to individual patient needs. Developing integrated data systems can be useful to improve data quality by linking clinical data sources such as hospital medical records to registries, ${ }^{106}$ or to create a comprehensive registry combining information from multiple registries. ${ }^{264}$

\section{Collaboration}

At times, national and international collaboration between registries may be needed to ensure an adequate sample size to study an outcome of interest, such as in the case of rare disease registries. Pregnancy registries in epilepsy, for example, require collaboration to identify a sufficient number of women exposed to various medications to examine occurrence of congenital malformations. ${ }^{265}$ Collaboration between registries through shared expertise and funding may also be a useful strategy to overcome challenges such as weak infrastructure, poor registry quality and insufficient coverage in a population. ${ }^{42}$ When combining registries, an integrated approach that supports an efficient exchange of information can minimize duplication and facilitate information exchange within the community. ${ }^{264}$ However, there are challenges associated with sustaining multijurisdictional registries including annual renewal for multiple centres, and multiple ethics review boards for the same registry. A national registry review board may help overcome these challenges. Internet-based registries and online data submission are feasible ways of gathering data from multiple countries. 103,259,262,263 Collaboration between state and federal registries is also effective for building national registries to ensure the data process is uniform and comparable. ${ }^{40}$

\section{Innovative Ways to Increase the Likelihood of a Feasible Registry}

In addition to the enabling factors described above, there are innovations that may be useful for increasing the likelihood of the implementation of a successful registry.

\section{Harmonization of Data Collection}

The EuroTARN group (http://eurotarn.man.ac.uk/) ${ }^{266}$ was established by several European collaborators and aimed to develop a common core dataset to assess the feasibility of collecting anonymous data as part of a trauma registry. ${ }^{263} \mathrm{~A}$ website that contained a new online data submission form was designed. To facilitate the creation of the dataset and consensus of opinions between contributors, the Delphi technique was used (views from the expert panel were collected through a series of online questionnaires). This online technique was beneficial for each stage of the technique to be completed on time and was less 
costly than meetings. The first stage involved asking participants to document as many clinical data points that they felt were necessary in the core dataset and document ideas for inclusion/exclusion. The second stage involved the categorization of and subsequent agreement ratings for all core data points submitted in stage one. The third stage involved voting on remaining core data points where an overwhelming rate of agreement was not achieved. The core dataset allows for the possibility to collect and combine outcome data in established trauma registries from representatives of 14 countries across Europe using a web-based system. It was successfully developed and trial data collection demonstrated the potential to collect clinical and epidemiological trauma data from a panEuropean perspective.

The National Institute for Neurological Disorders and Stroke initiated a common data elements project in order to streamline data collection for clinical research. The NINDS common data elements website (http://www.commondataelements.ninds.nih. gov/\#page $=$ Default $)^{267}$ serves as a repository of common data elements for clinical investigators. It provides access to NINDS common data elements definitions, as well as sample data collection forms. In addition to general common data elements, disease specific common data elements have been created for Amyotrophic Lateral Sclerosis, Congenital Muscular Dystrophy, Epilepsy, Friedrich's Ataxia, Multiple Sclerosis, Parkinson's Disease, Spinal Cord Injury, Stroke, and Traumatic Brain Injury.

\section{Timeliness of Data Reporting}

Since traditional sources of information (publications and presentations) lack timeliness in terms of recognition and reporting, Hauser et al established an Internet-based registry of pacemaker and ICD pulse generator and lead failures. ${ }^{262}$ This Internet-based registry could recognize and report device problems quickly. Quarterly data summaries are posted on the website and emailed to participants. When unexpected trends were observed, emailed alerts were issued to participants. Through the use of an Internet-based registry with data from multiple centers, important data can be transmitted in a timely manner. Registries may adopt such a system to report data quickly.

The Global Registry of Acute Coronary Events (GRACE) study tested the feasibility of a simplified data collection tool and provision of a quarterly feedback to index individual hospital management practices to an international reference cohort. ${ }^{268}$ They provided sequential, longitudinal data that enabled health care providers to identify potential care gaps, implement appropriate changes to the appropriate diagnostic management approach to patients with a suspected acute coronary syndrome, and measure the impact of changes on quality measures and clinical outcomes. GRACE successfully showed that individual hospitals can index their data management practices to an international reference cohort using simplified data collection tools. This allows health care providers to identify care gaps and potentially implement changes to diagnostic and management approaches.

To encourage researchers and clinicians to collaborate and share information for Disorders of Sex Development (DSD) in the European DSD registry, a web-based registry and virtual research environment (VRE) was developed. ${ }^{257}$ Consensus around a core data model was developed to eliminate heterogeneity in data. This VRE allows clinicians to enter data to assist researchers in finding eligible patients for study recruitment. This can allow for the collection of standardized data internationally, thereby, allowing collaborative research to be performed globally.

In certain instances, the existence of multiple independent registries on the same topic area may reduce their effectiveness. Non-collaborating registries in the same topic area result in the need for investigators to identify and visit several resources to obtain required information. Duplication amongst these resources will further complicate effective use of the registry data, impacting its overall value to the scientific community. ${ }^{264}$ One solution to this problem is for individual databases to be complementary and interlinked. ${ }^{264}$ This integrated approach could support efficient information exchange. Another possibility is the creation of a comprehensive registry that contains information currently provided by existing independent registries. ${ }^{264}$

\section{Other Considerations}

\section{Incentives for Patient Participation}

Some registries provide patients with small tokens to express appreciation for participating, for example, if a participants does not miss follow up sessions, they would be awarded "super participant" status, gold star, or a small gift such as an article of clothing with the registry logo on it. Incentives for patient participants such as newsletters, or compensation are particularly beneficial. The NARCOMS registry distributes a 20 page magazine, 'NARCOMS Now', to participants every three months (http://narcoms.org/narcomsnow/home) ${ }^{269}$; it provides participants with information about MS, recent research findings, and includes updates on the contributions of participants.

The ability to perform telephone or video-link follow-up visits or to do web-based follow-up visits may be beneficial for participants living in remote areas, and permission to perform various types of follow-up visits should be addressed during the initial consent process. Additional incentives such as: paying for parking when appropriate, giving community service points to those who need them to graduate from school, and/or giving small gifts such as movie passes may assist in retaining patients in a registry. 


\section{RECOMMENDATIONS}

A feasible registry with a high degree of impact will:

$\checkmark$ Have adequate advance planning and infrastructure (including human and monetary resources).

$\checkmark$ Incorporate minimal data collection time and frequency while tailoring the mode of data collection to participant needs.

$\checkmark$ Pilot test data collection practices to ensure they work as designed.

$\checkmark$ Have a diverse advisory board representing ethics, legal, operational, participant and sponsor interests.

$\checkmark$ Employ regular communication amongst all stakeholders.

$\checkmark$ Utilize graduated consent, and other participant retention tools such as a registry website and newsletter. $\checkmark$ Regularly engage providers through training meetings, regular data reports and presentations at conferences.

$\checkmark$ Cultivate long-term funding through activities that raise awareness about the importance of the registry.

$\checkmark$ Act in a transparent manner.

$\checkmark$ Utilize common data elements to enhance registry compatibility.

$\checkmark$ Link with vital statistics to determine whether patient has died and address other accessible information that may be of interest (seek patient consent for this).

$\checkmark$ Address challenges associated with recruitment and retention of members of minority groups to ensure representativeness. 